01

\title{
Корректность определения характеристик перемежающейся обобщенной синхронизации при использовании только одной переменной ведомой и вспомогательной систем
}

\author{
(C) А.А. Короновский, О.И. Москаленко, А.О. Сельский \\ Саратовский национальный исследовательский государственный университет им. Н.Г. Чернышевского, Саратов, Россия \\ ฯ E-mail: selskiiao@gmail.com
}

Поступило в Редакцию 21 ноября 2019г.

В окончательной редакции 9 января 2020г.

Принято к публикации 17 января 2020г.

\begin{abstract}
Рассмотрен метод выделения характерных фаз поведения в режиме перемежающейся обобщенной синхронизации, основанный на использовании вспомогательной системы. Установлено, что для корректного определения типа перемежаемости, реализуемого в системе, достаточно использовать только одну переменную, характеризующую состояние этой системы.
\end{abstract}

Ключевые слова: перемежающаяся обобщенная синхронизация, метод вспомогательной системы, фазы синхронного и асинхронного поведения.

DOI: 10.21883/PJTF.2020.07.49221.18126

Одним из наиболее интересных типов синхронного поведения связанных динамических систем является режим обобщенной хаотической синхронизации [1]. Этот режим может наблюдаться как в однонаправленно, так и во взаимно связанных системах и означает наличие функциональной связи (функционала) между их состояниями [1-3]. Интерес к этому режиму обусловлен как большим фундаментальным значением его исследования, так и возможностью его практического использования, например применения в задачах скрытой передачи информации [4].

Для диагностики режима обобщенной синхронизации предложены различные методы и подходы, среди которых наибольшее распространение получил метод вспомогательной системы [5]. Согласно этому подходу, дающему корректные результаты только в случае однонаправленной связи, наряду с исходными ведущей и ведомой системами в рассмотрение вводится еще и вспомогательная система, идентичная ведомой системе по управляющим параметрам, но стартующая с других начальных условий. Если после завершения переходного процесса состояния ведомой и вспомогательной систем станут идентичными, можно говорить о наличии обобщенной синхронизации между ведущим и ведомым осцилляторами.

На границе обобщенной синхронизации наблюдается, как правило, перемежающееся поведение - перемежающаяся обобщенная синхронизация [6], определить характеристики которой можно также при помощи метода вспомогательной системы. Для этого необходимо рассмотреть разность между состояниями ведомой и вспомогательной систем, которая в данном случае будет представлять собой чередование участков с синхронной (ламинарные фазы) и асинхронной (турбулентные фазы) динамикой, и определить длительности тех интервалов, где наблюдается синхронное поведение. Получив разумную статистику длительностей ламинарных фаз, можно определить тип перемежаемости, наблюдаемый в системе. Известно, что на границе обобщенной синхронизации наблюдается перемежаемость типа ,on-off' [6].

При применении метода вспомогательной системы традиционно рассматривается разность между состояниями ведомой и вспомогательной систем только по одной переменной. Такой исторически сложившийся подход достаточно удобен при обработке экспериментальных временны́х рядов [7], когда нет возможности получить информацию о других переменных (например, возможно снять напряжение только с одного участка цепи), при анализе пространственно-распределенных систем или систем с запаздыванием, характеризующихся бесконечномерным фазовым пространством (см., например, [8,9]). При анализе сосредоточенных автоколебательных систем также, как правило, используется только одна переменная в связи с простотой произведения расчетов. В то же время понятно, что во всех случаях более корректно использовать все переменные, входящие в состояние системы.

В настоящей работе на примере двух однонаправленно связанных сосредоточенных систем, находящихся в режиме перемежающейся обобщенной синхронизации, рассматривается вопрос о целесообразности и корректности определения характеристик этого режима с помощью использования только одной переменной. Как показано далее, учет всех координат оказывается избыточным и не приводит к существенным изменениям статистических характеристик перемежаемости. 

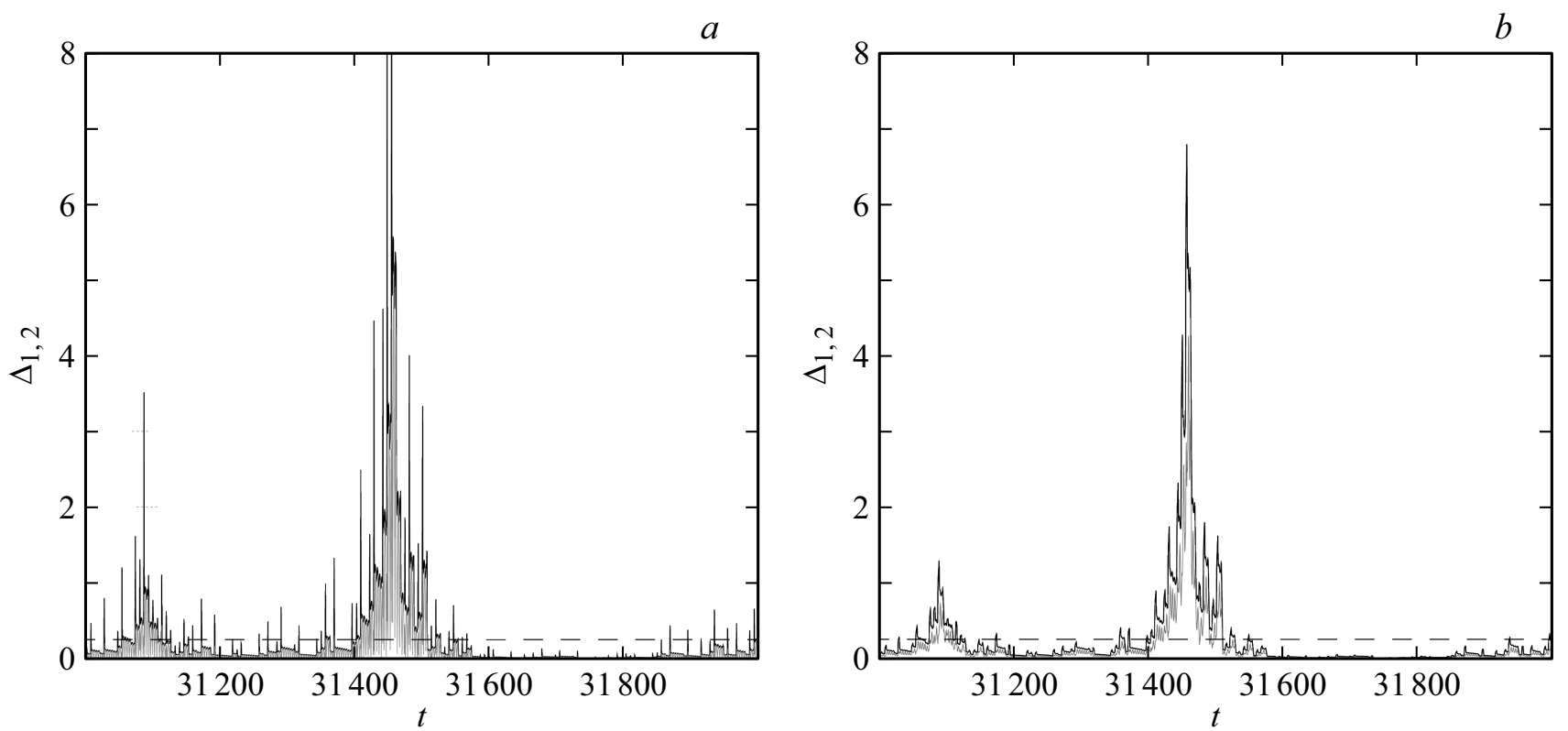

Рис. 1. $a$ - зависимости разности состояний ведомой и вспомогательной систем Ресслера (1) от времени при значении параметра связи $\varepsilon=0.17$, рассчитанные по переменной $x$ (серые линии) и по эвклидовой мере трех фазовых переменных (черные линии). Штриховой линией показан порог определения турбулентной фазы. $b-$ аналогичные зависимости, полученные с применением скользящего среднего (интервал усреднения $\tau=2.2$ ).

В качестве объекта исследования выбраны две однонаправленно связанные системы Ресслера [10]:

$$
\begin{gathered}
\dot{x}_{d}=-\omega_{d} y_{d}-z_{d}, \\
\dot{y}_{d}=\omega_{d} x_{d}+a y_{d}, \\
\dot{z}_{d}=p+z_{d}\left(x_{d}-c\right), \\
\dot{x}_{r}=-\omega_{r} y_{r}-z_{r}+\varepsilon\left(x_{d}-x_{r}\right), \\
\dot{y}_{r}=\omega_{r} x_{r}+a y_{r}, \\
\dot{z}_{r}=p+z_{r}\left(x_{r}-c\right),
\end{gathered}
$$

где $a=0.15, p=0.2, c=10, \omega_{d}=0.95, \omega_{r}=0.93-$ управляющие параметры, $\varepsilon-$ параметр связи.

Для диагностики обобщенной синхронизации в системе (1) дополнительно введена в рассмотрение вспомогательная система

$$
\begin{gathered}
\dot{x}_{a}=-\omega_{a} y_{a}-z_{a}+\varepsilon\left(x_{d}-x_{a}\right), \\
\dot{y}_{a}=\omega_{a} x_{a}+a y_{a}, \\
\dot{z}_{a}=p+z_{a}\left(x_{a}-c\right) .
\end{gathered}
$$

Установлено [11], что режим обобщенной синхронизации возникает в данном случае при $\varepsilon_{c}=0.178$, ниже границы синхронного режима диагностируется перемежающееся поведение.

На рис. 1, $a$ приведены зависимости разности состояний ведомой и вспомогательной систем от времени в режиме перемежаемости, полученные при значении параметра связи $\varepsilon=0.17$ по одной переменной

$$
\Delta_{1}=\left|x_{a}-x_{r}\right|
$$

(серые линии) и по всем трем переменным

$$
\Delta_{2}=\sqrt{\left(x_{a}-x_{r}\right)^{2}+\left(y_{a}-y_{r}\right)^{2}+\left(z_{a}-z_{r}\right)^{2}}
$$

(черные линии). Штриховой линией показано значение порога, условно разделяющего ламинарные и турбулентные фазы. Из рисунка видно, что в обоих случаях заметны недостатки. При введении разницы состояний по переменной $x$ значение величины $\Delta_{1}$ очень быстро изменяется со временем. В итоге при значениях $\Delta_{1}$, близких к порогу, будет выделено много очень коротких ламинарных фаз, что ухудшает статистику и требует дополнительной обработки результатов. При введении разности состояний по трем координатам у величины $\Delta_{2}$ возникают резкие изменения, связанные с динамикой переменной $z$. Такие пики величины $\Delta_{2}$ также приводят к тому, что некоторые ламинарные фазы оказываются разбитыми на несколько „фаз“, что занижает длительности ламинарных фаз и увеличивает их количество. Недостатки обоих подходов можно уменьшить, если использовать скользящее среднее, как показано на рис. $1, b$.

При помощи обоих подходов (выделение ламинарных фаз по одной и по всем переменным), описанных выше, для скользящего среднего были получены распределения длительностей ламинарных фаз при различных значениях параметра связи, которые приведены на рис. 2 . Серый цвет соответствует случаю использования только одной переменной $x$, черный - всех переменных. Распределения были нормированы на общее число ламинар- 

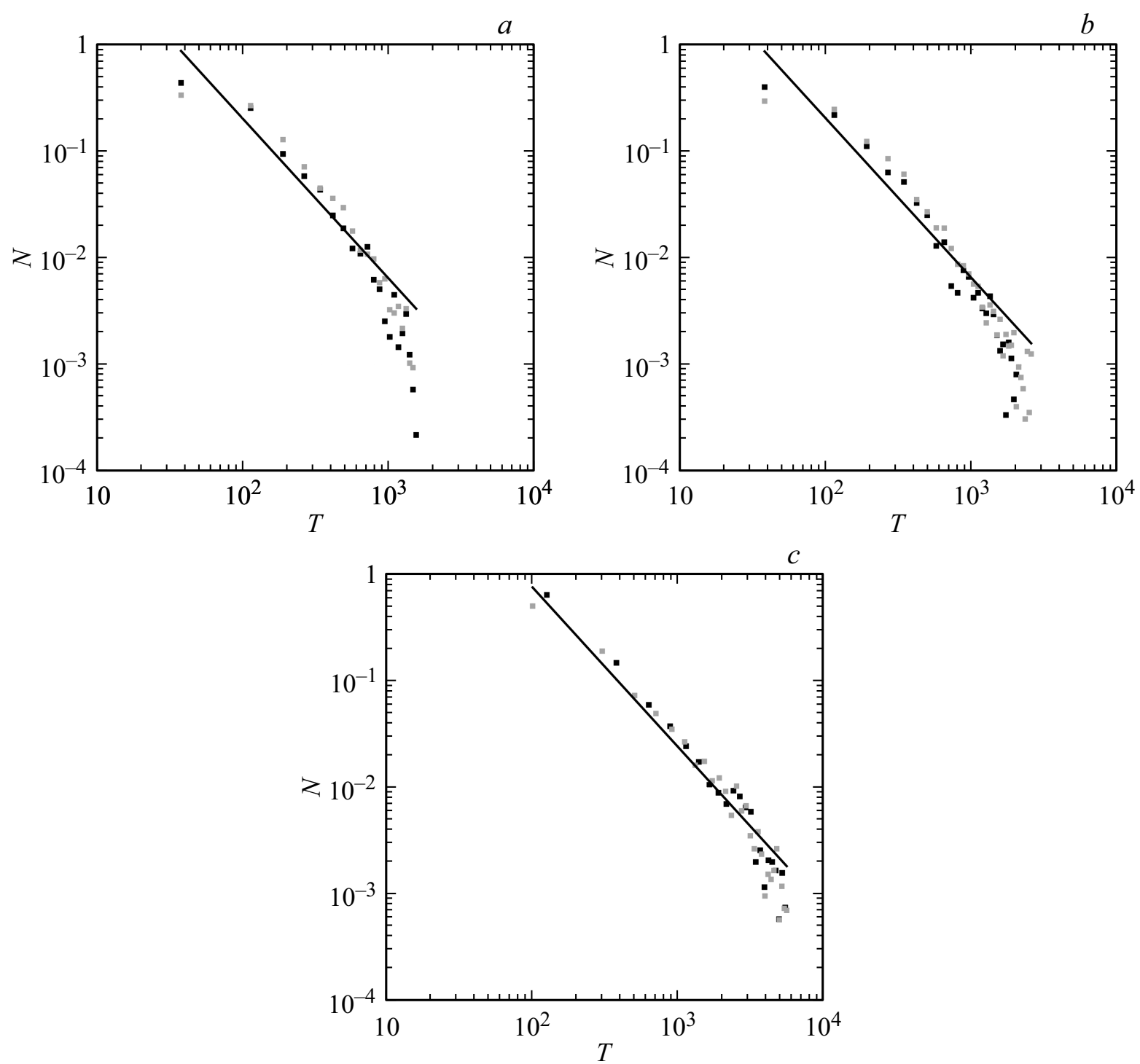

Рис. 2. Распределения длительностей ламинарных фаз, нормированные на общее число фаз, рассчитанные по переменной $x$ (серые точки) и по эвклидовой мере трех фазовых переменных (черные точки), для трех значений параметра связи: $\varepsilon=0.15$ ( $a$ ), 0.16 $(b), 0.17(c)$. Для всех значений параметров число ламинарных фаз превышало 2000. Линией показана аппроксимация степенным законом (5): $a=200(a, b)$ и $750(c)$.

ных фаз в каждом случае. Результаты были аппроксимированы степенным законом

$$
N=a T^{-3 / 2},
$$

что соответствует теоретической закономерности, характерной для „оn-off“-перемежаемости [6,12]. Из рис. 2 видно, что во всех рассмотренных случаях результаты численного моделирования находятся в хорошем согласии с теоретической закономерностью. Более того, полученные результаты качественно не отличаются друг от друга при использовании двух различных подходов для выделения ламинарных фаз. Можно ожидать аналогичных результатов при проведении расчетов для экспериментальных данных и систем с бесконечномерным фазовым пространством, и соответственно при подобных исследованиях достаточно учитывать только одну из переменных системы.

\section{Финансирование работы}

Работа выполнена при финансовой поддержке Российского научного фонда (проект № 19-12-00037).

\section{Конфликт интересов}

Авторы заявляют, что у них нет конфликта интересов. 


\section{Список литературы}

[1] Rulkov N.F., Sushchik M.M., Tsimring L.S., Abarbanel H.D.I. // Phys. Rev. E. 1995. V. 51. N 2. P. 980-994. DOI: 10.1103/PhysRevE.51.980

[2] Koronovskii A.A., Moskalenko O.I., Hramov A.E. // Phys. Rev. E. 2011. V. 84. N 3. P. 037201.

DOI: 10.1103/PhysRevE.84.037201

[3] Moskalenko O.I., Koronovskii A.A., Hramov A.E., Boccaletti S. // Phys. Rev. E. 2012. V. 86. N 3. P. 036216. DOI: 10.1103/PhysRevE.86.036216

[4] Короновский А.А., Москаленко О.И., Храмов А.Е. // УФН. 2009. T. 179. В. 12. C. $1281-1310$.

DOI: $10.3367 /$ UFNr.0179.200912c.1281

[5] Abarbanel H.D.I., Rulkov N.F., Sushchik M. // Phys. Rev. E. 1996. V. 53. N 5. P. $4528-4535$.

DOI: 10.1103/PhysRevE.53.4528

[6] Hramov A.E., Koronovskii A.A. // Europhys. Lett. 2005. V. 70. N 2. P. $169-175$. DOI: $10.1209 / \mathrm{epl} / \mathrm{i} 2004-10488-6$

[7] Овчинников А.А., Москаленко О.И., Короновский А.А., Храмов А.Е. // Письма в ЖТФ. 2010. Т. 36. В. 4. С. 1-7.

[8] Попов П.В. // Письма в ЖТФ. 2007. Т. 33. В. 18. С. 61-69.

[9] Плотникова А.Д., Москаленко О.И. // Письма в ЖТФ. 2019. T. 45. B. 11. C. 31-33.

[10] Куровская М.К. // Письма в ЖТФ. 2008. Т. 34. В. 24. C. $48-54$.

[11] Hramov A.E., Koronovskii A.A., Moskalenko O.I. // Europhys. Lett. 2005. V. 72. N 6. P. 901-907.

DOI: $10.1209 / \mathrm{epl} / \mathrm{i} 2005-10343-4$

[12] Boccaletti S., Valladares D.L. // Phys. Rev. E. 2000. V. 62. N 5. P. 7497-7500. 